\title{
Thermal and vacuum friction acting on rotating particles
}

\author{
A. Manjavacas and F. J. García de Abajo* \\ Instituto de Óptica - CSIC, Serrano 121, E-28006 Madrid, Spain
}

(Received 5 October 2010; published 23 December 2010)

\begin{abstract}
We study the stopping of spinning particles in vacuum. A torque is produced by fluctuations of the vacuum electromagnetic field and the particle polarization. Expressions for the frictional torque and the power radiated by the particle are obtained as a function of rotation velocity and the temperatures of the particle and the surrounding vacuum. We solve this problem following two different approaches: (i) a semiclassical calculation based upon the fluctuation-dissipation theorem (FDT), and (ii) a fully quantum-mechanical theory within the framework of quantum electrodynamics, assuming that the response of the particle is governed by bosonic excitations such as phonons and plasmons. Both calculations lead to identical final expressions, thus confirming the suitability of the FDT to deal with problems that are apparently out of equilibrium, and also providing comprehensive insight into the physical processes underlying thermal and vacuum friction. We adapt the quantum-mechanical theory to describe particles whose electromagnetic response is produced by fermionic excitations. Furthermore, we extend our FDT formalism to fully account for magnetic polarization, which dominates friction when the particle is a good conductor. Finally, we present numerically calculated torques and stopping times for the relevant cases of graphite and gold nanoparticles.
\end{abstract}

DOI: 10.1103/PhysRevA.82.063827

PACS number(s): 42.50.Wk, 41.60.-m, 45.20.dc, 78.70.-g

\section{INTRODUCTION}

The absoluteness of rotational motion, first demonstrated by Newton's bucket experiment, emerges in numerous electrodynamic phenomena, for example, through interferometry (Sagnac effect [1]) and light emission (rotational frequency shift [2,3]). Likewise, the rotation of charged objects (e.g., electric [4] and magnetic [5] dipoles) produces radiation emission, and as a result, also reaction torques [6]. More surprisingly, the angular momentum carried by light can be transformed into mechanical rotation of neutral particles $[7,8]$, so the question arises, is the reverse possible? Does a homogeneous, neutral particle emit light simply by rotating?

Accelerated neutral objects are known to produce so-called Casimir radiation by dynamical changes in the boundary conditions of the electromagnetic fields associated to photon states [9,10]. A similar effect is at the origin of noncontact friction between objects set in relative uniform motion $[11,12]$. The case of two planar homogeneous surfaces, which has recently generated a heated debate [13], is particularly striking because no perceptible changes in physical boundaries are associated to parallel relative displacements. A related instance is a spinning sphere, in which vacuum and thermal friction would constitute a strong test of the absolute character of rotational motion.

The study of such a rotating system is the main objective of this paper. In particular, we investigate the friction experienced by rotating, neutral particles due to the interaction with the vacuum and thermal electromagnetic field. For this purpose we first follow a semiclassical approach as sketched in a previous publication [14], based upon the fluctuation-dissipation theorem (FDT) [15,16]. This formalism allows us to find analytical expressions for both the frictional torque and the power radiated by the particle. Although the semiclassical

\footnotetext{
*Author to whom correspondence should be addressed. J.G.deAbajo@csic.es
}

model is completely rigorous and unambiguous, one could question the validity of the FDT for a system that is out of mechanical equilibrium. In order to clarify this point, we develop a fully quantum-mechanical (QM) theory, in which the electromagnetic response of the particle is governed by bosonic excitations such as phonons and plasmons. The results of both the semiclassical and the QM approaches are in complete agreement, thus allowing us to obtain deeper understanding of the physical processes underlying thermal and vacuum friction. For instance, the QM approach provides us with a solid argument to predict, within the limits of our model, the absence of friction in nonabsorbing spherical particles possessing a large optical gap compared to the rotation and the thermal-radiation frequencies. In contrast, particles made of lossy materials such as metals undergo friction even at zero temperature, unlike what has been previously predicted [17].

This paper is organized as follows. We derive the thermal and vacuum friction from the semiclassical FDT approach in Sec. II. A fully QM approach is presented and developed in Sec. III for a particle characterized by bosonic excitations. Section IV is devoted to showing the equivalence of both approaches. The quantum theory is extended in Sec. V to deal with fermionic excitations, such as those of a twolevel molecule. In Sec. VI, we supplement our semiclassical formalism to incorporate magnetic polarization, and we offer a comparison of the relative contributions of electric and magnetic responses. Numerical results of the torque and the stopping time are given for graphite and gold nanoparticles. Finally, the main conclusions are summarized in Sec. VII. We use Gaussian electromagnetic units, unless otherwise stated.

\section{FLUCTUATION-DISSIPATION-THEOREM APPROACH}

We consider the system described in Fig. 1, which consists of an isotropic particle at temperature $T_{1}$ placed in vacuum at temperature $T_{0}$. The particle is rotating around its $z$ axis with 


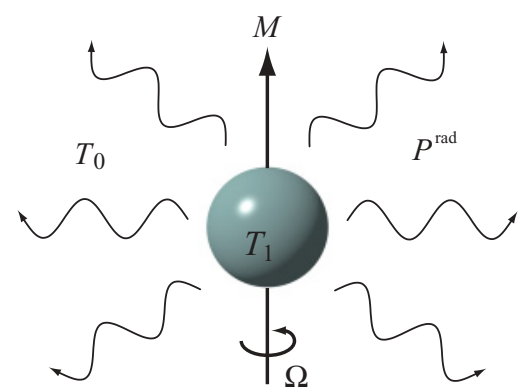

FIG. 1. (Color online) Description of the system under study. The particle is at temperature $T_{1}$ and rotates with angular frequency $\Omega$ around the $z$ axis. The surrounding vacuum is at temperature $T_{0}$. The particle experiences a torque $M$ and emits a power $P^{\text {rad }}$ in the form of radiation.

angular frequency $\Omega$ and interacts with the radiation field in its surroundings. This interaction produces a torque $M$ on the particle, and it gives rise to radiation with a net power $P^{\mathrm{rad}}$. The particle radius $a$ is assumed to be small compared to the wavelength of the involved radiation, which is controlled by the temperatures $T_{0}$ and $T_{1}$ (through the thermal radiation profile) and the rotation frequency $\Omega$. This approximation implies that both $\Omega a / c$ and $k_{B} T_{j} a / c \hbar$ are small compared to unity [18], thus allowing us to describe the electromagnetic response of the particle via its frequency-dependent dipolar polarizability.

\section{A. Frictional torque}

In the calculation of the torque acting on a rotating particle we have to include two separate contributions: (i) the fluctuations of the particle dipole, and (ii) the fluctuations of the electromagnetic field. The torque produced by an electric field $\mathbf{E}$ on a dipole $\mathbf{p}$ is simply given by $\mathbf{p} \times \mathbf{E}$. We can use this result to write the torque on our particle along the rotation axis $\hat{\mathbf{z}}$ as

$$
M=\left\langle\mathbf{p}(t) \times \mathbf{E}\left(\mathbf{r}_{0}, t\right)\right\rangle \cdot \hat{\mathbf{z}}
$$

where \langle\rangle represents the average over fluctuations and the field is evaluated at the position of the particle $\mathbf{r}=\mathbf{r}_{0}$. We can separate the two contributions just described in the following way:

$$
\begin{aligned}
M & =\left\langle\mathbf{p}^{\mathrm{fl}}(t) \times \mathbf{E}^{\text {ind }}\left(\mathbf{r}_{0}, t\right)+\mathbf{p}^{\text {ind }}(t) \times \mathbf{E}^{\mathrm{fl}}\left(\mathbf{r}_{0}, t\right)\right\rangle \cdot \hat{\mathbf{z}} \\
& =M_{p}+M_{E} .
\end{aligned}
$$

The first term accounts for the fluctuations of the particle dipole that correlate with the resulting induced field, while the second one involves field fluctuations and the dipole that they induce. There are no cross terms involving fluctuations of both the dipole and the field because they belong to different physical systems, so that they are uncorrelated. It is convenient to work in frequency space $\omega$, defined via the Fourier transform

$$
\mathbf{E}(t)=\frac{1}{2 \pi} \int_{-\infty}^{\infty} d \omega \mathbf{E}(\omega) e^{-i \omega t}
$$

for the electric field, and similarly for other quantities. This allows us to express the induced field of Eq. (1) in terms of the fluctuating dipole by using the electromagnetic Green tensor $G$ :

$$
\mathbf{E}^{\text {ind }}(\mathbf{r}, \omega)=G\left(\mathbf{r}, \mathbf{r}_{0}, \omega\right) \cdot \mathbf{p}^{\mathrm{fl}}(\omega) .
$$

The Cartesian components of $G$ in vacuum are given by

$$
\begin{aligned}
G_{i j}\left(\mathbf{r}, \mathbf{r}_{0}, \omega\right)= & \frac{\exp (i k R)}{R^{3}}\left[\left(k^{2} R^{2}+i k R-1\right) \delta_{i j}\right. \\
& \left.-\left(k^{2} R^{2}+3 i k R-3\right) \frac{R_{i} R_{j}}{R^{2}}\right]
\end{aligned}
$$

where $\mathbf{R}=\mathbf{r}-\mathbf{r}_{0}$ and $k=\omega / c$. In a similar way, the induced dipole can be written in terms of the fluctuating field with the help of the particle polarizability tensor,

$$
\mathbf{p}^{\text {ind }}(\omega)=\alpha(\omega) \cdot \mathbf{E}^{\mathrm{fl}}\left(\mathbf{r}_{0}, \omega\right)
$$

For simplicity, we assume particles with axial symmetry, for which the nonvanishing components of the polarizability tensor can be written as

$$
\begin{gathered}
\alpha_{x x}(\omega)=\alpha_{y y}(\omega)=\alpha_{\perp}(\omega), \\
\alpha_{z z}(\omega)=\alpha_{\|}(\omega) .
\end{gathered}
$$

These expressions, together with Eq. (1), permit us to write the torque as the sum of two terms, one of them quadratic with respect to the fluctuating dipole and the other one quadratic with respect to the fluctuating field. These quadratic terms have to be averaged over fluctuations using the symmetrized version of the FDT [14-16], which in our case reads

$$
\begin{aligned}
\left\langle p_{i}^{\mathrm{fl}}(\omega) p_{j}^{\mathrm{fl}}\left(\omega^{\prime}\right)\right\rangle= & 4 \pi \hbar \delta\left(\omega+\omega^{\prime}\right) \\
& \times \operatorname{Im}\left\{\alpha_{i j}(\omega)\right\}\left(n_{1}(\omega)+\frac{1}{2}\right)
\end{aligned}
$$

for the dipole fluctuations and

$$
\begin{aligned}
\left\langle E_{i}^{\mathrm{fl}}(\mathbf{r}, \omega) E_{j}^{\mathrm{fl}}\left(\mathbf{r}^{\prime}, \omega^{\prime}\right)\right\rangle= & 4 \pi \hbar \delta\left(\omega+\omega^{\prime}\right) \\
& \times \operatorname{Im}\left\{G_{i j}\left(\mathbf{r}, \mathbf{r}^{\prime}, \omega\right)\right\}\left(n_{0}(\omega)+\frac{1}{2}\right)
\end{aligned}
$$

for the electric-field fluctuations. The temperatures of the particle $\left(T_{1}\right)$ and the vacuum $\left(T_{0}\right)$ enter these expressions through the Bose-Einstein distribution functions

$$
n_{l}(\omega)=\frac{1}{e^{\hbar \omega / k_{B} T_{l}}-1} .
$$

We first calculate the term associated to the dipole fluctuations,

$$
\begin{aligned}
M_{p} & =\left\langle\mathbf{p}^{\mathrm{fl}}(t) \times \mathbf{E}^{\mathrm{ind}}\left(\mathbf{r}_{0}, t\right)\right\rangle \cdot \mathbf{z} \\
& =\int_{-\infty}^{\infty} \frac{d \omega d \omega^{\prime}}{(2 \pi)^{2}} e^{-i\left(\omega+\omega^{\prime}\right) t}\left\langle\mathbf{p}^{\mathrm{fl}}(\omega) \times \mathbf{E}^{\text {ind }}\left(\mathbf{r}_{0}, \omega^{\prime}\right)\right\rangle \cdot \mathbf{z} .
\end{aligned}
$$

Using Eq. (2) to write the induced field in terms of the fluctuating dipole, Eq. (7) becomes

$$
\begin{aligned}
M_{p}= & \int_{-\infty}^{\infty} \frac{d \omega d \omega^{\prime}}{(2 \pi)^{2}} e^{-i\left(\omega+\omega^{\prime}\right) t}\left[p_{x}^{\mathrm{fl}}(\omega) G_{y x}\left(\mathbf{r}_{0}, \mathbf{r}_{0}, \omega^{\prime}\right) p_{x}^{\mathrm{fl}}\left(\omega^{\prime}\right)\right. \\
& +p_{x}^{\mathrm{fl}}(\omega) G_{y y}\left(\mathbf{r}_{0}, \mathbf{r}_{0}, \omega^{\prime}\right) p_{y}^{\mathrm{fl}}\left(\omega^{\prime}\right)-p_{y}^{\mathrm{fl}}(\omega) G_{x x}\left(\mathbf{r}_{0}, \mathbf{r}_{0}, \omega^{\prime}\right) \\
& \left.\times p_{x}^{\mathrm{fl}}\left(\omega^{\prime}\right)-p_{y}^{\mathrm{fl}}(\omega) G_{x y}\left(\mathbf{r}_{0}, \mathbf{r}_{0}, \omega^{\prime}\right) p_{y}^{\mathrm{fl}}\left(\omega^{\prime}\right)\right]
\end{aligned}
$$

So far we have expressed the fluctuating dipoles in the laboratory frame (see Fig. 2), but we need to transform them to the rotating frame in order to average over fluctuations 


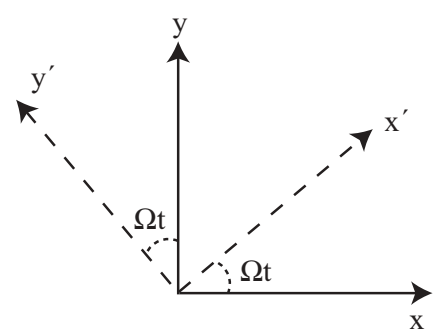

FIG. 2. Definition of the rotating frame (dashed lines) and the laboratory frame (solid lines). The former rotates with angular frequency $\Omega$.

via the FDT. The physical reason for this is that the particle polarizability can only be applied in the rotating frame, in which the electronic and vibrational excited states that produce polarization are well defined. Furthermore, we assume that those states are unaffected by the rotational motion in the rest frame of the particle. We can express the laboratory-frame dipoles $\mathbf{p}$ in terms of the dipoles in the rotating frame $\mathbf{p}^{\prime}$ in the following way:

$$
\begin{gathered}
p_{x}(\omega)=\frac{1}{2}\left[p_{x}^{\prime}\left(\omega_{+}\right)+i p_{y}^{\prime}\left(\omega_{+}\right)+p_{x}^{\prime}\left(\omega_{-}\right)-i p_{y}^{\prime}\left(\omega_{-}\right)\right], \\
p_{y}(\omega)=\frac{1}{2}\left[-i p_{x}^{\prime}\left(\omega_{+}\right)+p_{y}^{\prime}\left(\omega_{+}\right)+i p_{x}^{\prime}\left(\omega_{-}\right)+p_{y}^{\prime}\left(\omega_{-}\right)\right], \\
p_{z}(\omega)=p_{z}^{\prime}(\omega),
\end{gathered}
$$

where we have employed the compact notation $\omega_{ \pm}=\omega \pm \Omega$. Introducing Eq. (9) into Eq. (8) and averaging over fluctuations via the FDT [Eq. (4)], we obtain the expression

$$
\begin{aligned}
M_{p}= & \frac{i \hbar}{2 \pi} \int_{-\infty}^{\infty} d \omega d \omega^{\prime} e^{-i\left(\omega+\omega^{\prime}\right) t} \\
& \times\left[G_{x x}\left(\mathbf{r}_{0}, \mathbf{r}_{0}, \omega^{\prime}\right)+G_{y y}\left(\mathbf{r}_{0}, \mathbf{r}_{0}, \omega^{\prime}\right)\right] \\
& \times\left[\delta\left(\omega_{+}+\omega_{-}^{\prime}\right) \operatorname{Im}\left\{\alpha_{\perp}\left(\omega_{+}\right)\right\}\left(n_{1}\left(\omega_{+}\right)+\frac{1}{2}\right)\right. \\
& \left.-\delta\left(\omega_{-}+\omega_{+}^{\prime}\right) \operatorname{Im}\left\{\alpha_{\perp}\left(\omega_{-}\right)\right\}\left(n_{1}\left(\omega_{-}\right)+\frac{1}{2}\right)\right] .
\end{aligned}
$$

Now, performing the integral over $\omega^{\prime}$, taking into account causality in the Green tensor [i.e., $G\left(\mathbf{r}, \mathbf{r}^{\prime},-\omega\right)=$ $\left.G^{*}\left(\mathbf{r}, \mathbf{r}^{\prime}, \omega\right)\right]$, and noticing that $n_{l}(\omega)+1 / 2$ is an odd function of $\omega$, we find

$$
\begin{aligned}
M_{p}= & \frac{\hbar}{\pi} \int_{0}^{\infty} d \omega \operatorname{Im}\left\{G_{x x}\left(\mathbf{r}_{0}, \mathbf{r}_{0}, \omega\right)+G_{y y}\left(\mathbf{r}_{0}, \mathbf{r}_{0}, \omega\right)\right\} \\
& \times\left[\operatorname{Im}\left\{\alpha_{\perp}\left(\omega_{+}\right)\right\}\left(n_{1}\left(\omega_{+}\right)+\frac{1}{2}\right)\right. \\
& \left.-\operatorname{Im}\left\{\alpha_{\perp}\left(\omega_{-}\right)\right\}\left(n_{1}\left(\omega_{-}\right)+\frac{1}{2}\right)\right]
\end{aligned}
$$

Now, from the definition of the Green tensor, one has

$$
\lim _{\left|\mathbf{r}-\mathbf{r}^{\prime}\right| \rightarrow 0} \operatorname{Im}\left\{G_{i j}\left(\mathbf{r}, \mathbf{r}^{\prime}, \omega\right)\right\}=\frac{2}{3} \frac{\omega^{3}}{c^{3}} \delta i j,
$$

and using this expression in Eq. (10) we obtain

$$
\begin{aligned}
M_{p}= & \frac{4 \hbar}{3 \pi c^{3}} \int_{0}^{\infty} d \omega \omega^{3}\left[\operatorname{Im}\left\{\alpha_{\perp}\left(\omega_{+}\right)\right\}\left(n_{1}\left(\omega_{+}\right)+\frac{1}{2}\right)\right. \\
& \left.-\operatorname{Im}\left\{\alpha_{\perp}\left(\omega_{-}\right)\right\}\left(n_{1}\left(\omega_{-}\right)+\frac{1}{2}\right)\right] .
\end{aligned}
$$

Equation (12) only contains the torque produced due to electric-dipole fluctuations. Coming back to Eq. (1), we still have to calculate its second term, which accounts for the torque associated to field fluctuations:

$$
\begin{aligned}
M_{E} & =\left\langle\mathbf{p}^{\text {ind }}(t) \times \mathbf{E}^{\mathrm{fl}}(\mathbf{r}, t)\right\rangle \cdot \mathbf{z} \\
& =\int_{-\infty}^{\infty} \frac{d \omega d \omega^{\prime}}{(2 \pi)^{2}} e^{-i\left(\omega+\omega^{\prime}\right) t} \mathbf{p}^{\text {ind }}(\omega) \times \mathbf{E}^{\mathrm{fl}}\left(\mathbf{r}, \omega^{\prime}\right) \cdot \mathbf{z} .
\end{aligned}
$$

In order to express the induced dipole in terms of the fluctuating field, we need to write the dipole in the rotating frame before we can apply Eq. (3), and then go back to the laboratory frame. This procedure leads to an effective polarizability for the rotating particle (see the Appendix), the components of which are

$$
\begin{gathered}
\alpha_{x x}^{\mathrm{eff}}(\omega)=\alpha_{y y}^{\mathrm{eff}}(\omega)=\frac{1}{2}\left[\alpha_{\perp}\left(\omega_{+}\right)+\alpha_{\perp}\left(\omega_{-}\right)\right], \\
\alpha_{x y}^{\mathrm{eff}}(\omega)=-\alpha_{y x}^{\mathrm{eff}}(\omega)=\frac{i}{2}\left[\alpha_{\perp}\left(\omega_{+}\right)-\alpha_{\perp}\left(\omega_{-}\right)\right], \\
\alpha_{z z}^{\mathrm{eff}}(\omega)=\alpha_{\|}(\omega) .
\end{gathered}
$$

Using this effective polarizability, Eq. (13) becomes

$$
\begin{aligned}
M_{E}= & \int_{-\infty}^{\infty} \frac{d \omega d \omega^{\prime}}{(2 \pi)^{2}} e^{-i\left(\omega+\omega^{\prime}\right) t}\left[\alpha_{x x}^{\mathrm{eff}}(\omega) E_{x}^{\mathrm{fl}}(\mathbf{r}, \omega) E_{y}^{\mathrm{fl}}\left(\mathbf{r}, \omega^{\prime}\right)\right. \\
& +\alpha_{x y}^{\mathrm{eff}}(\omega) E_{y}^{\mathrm{fl}}(\mathbf{r}, \omega) E_{y}^{\mathrm{fl}}\left(\mathbf{r}, \omega^{\prime}\right)-\alpha_{y x}^{\mathrm{eff}}(\omega) E_{x}^{\mathrm{fl}}(\mathbf{r}, \omega) E_{x}^{\mathrm{fl}}\left(\mathbf{r}, \omega^{\prime}\right) \\
& \left.-\alpha_{y y}^{\mathrm{eff}}(\omega) E_{y}^{\mathrm{fl}}(\mathbf{r}, \omega) E_{x}^{\mathrm{fl}}\left(\mathbf{r}, \omega^{\prime}\right)\right] .
\end{aligned}
$$

This expression is already prepared to perform the average over field fluctuations using the FDT [Eq. (5)]. We find

$$
\begin{aligned}
M_{E}= & \frac{2 \hbar}{3 \pi c^{3}} \int_{-\infty}^{\infty} d \omega d \omega^{\prime} e^{-i\left(\omega+\omega^{\prime}\right) t} \omega^{3}\left[\alpha_{x y}^{\text {eff }}(\omega)-\alpha_{y x}^{\text {eff }}(\omega)\right] \\
& \times \delta\left(\omega+\omega^{\prime}\right)\left(n_{0}(\omega)+\frac{1}{2}\right)
\end{aligned}
$$

where we have also used Eq. (11). Now we can perform the integral over $\omega^{\prime}$. Using the causality property of the polarizability $\alpha(-\omega)=\alpha^{*}(\omega)$ and the parity of the rest of the integrand in Eq. (15), we obtain

$$
\begin{aligned}
M_{E}= & \frac{4 \hbar}{3 \pi c^{3}} \int_{0}^{\infty} d \omega \omega^{3}\left[\operatorname{Im}\left\{\alpha_{\perp}\left(\omega_{-}\right)\right\}-\operatorname{Im}\left\{\alpha_{\perp}\left(\omega_{+}\right)\right\}\right] \\
& \times\left(n_{0}(\omega)+\frac{1}{2}\right)
\end{aligned}
$$

Finally, the total torque resulting from the sum of Eqs. (12) and (16) reduces to

$$
\begin{aligned}
M= & -\frac{4 \hbar}{3 \pi c^{3}} \int_{0}^{\infty} d \omega \omega^{3} \operatorname{Im}\left\{\alpha_{\perp}\left(\omega_{-}\right)\right\}\left[n_{1}\left(\omega_{-}\right)-n_{0}(\omega)\right] \\
& +\frac{4 \hbar}{3 \pi c^{3}} \int_{0}^{\infty} d \omega \omega^{3} \operatorname{Im}\left\{\alpha_{\perp}\left(\omega_{+}\right)\right\}\left[n_{1}\left(\omega_{+}\right)-n_{0}(\omega)\right] .
\end{aligned}
$$

A more compact expression can be found by playing with the integration limits:

$M=-\frac{4 \hbar}{3 \pi c^{3}} \int_{-\infty}^{\infty} d \omega \omega^{3} \operatorname{Im}\left\{\alpha_{\perp}\left(\omega_{-}\right)\right\}\left[n_{1}\left(\omega_{-}\right)-n_{0}(\omega)\right]$.

This expression makes it clear that, as expected, the torque vanishes for nonrotating nanoparticles. 
The generalization of Eq. (18) to the case of anisotropic particles with principal axes along $x, y$, and $z$ [i.e., $\alpha_{x x}(\omega) \neq$ $\left.\alpha_{y y}(\omega)\right]$ is straightforward, but it involves lengthy algebraic manipulations. We find

$$
\begin{aligned}
M= & -\frac{4 \hbar}{3 \pi c^{3}} \int_{-\infty}^{\infty} d \omega \omega^{3} \frac{1}{2} \operatorname{Im}\left\{\alpha_{x x}\left(\omega_{-}\right)+\alpha_{y y}\left(\omega_{-}\right)\right\} \\
& \times\left[n_{1}\left(\omega_{-}\right)-n_{0}(\omega)\right]
\end{aligned}
$$

and hence Eq. (18) is recovered, but we find $\alpha_{\perp}(\omega)$ as the average of the polarizabilities along the $x$ and $y$ axes.

\section{B. Net radiated power}

The interaction between the rotating particle and the surrounding field involves photon exchanges that lead to a net radiated power. We calculate this power here following a procedure similar to the one used for the torque. First we write

$$
\begin{aligned}
P^{\mathrm{rad}} & =-\left\langle\mathbf{E}^{\mathrm{ind}}(\mathbf{r}, t) \cdot \frac{\partial \mathbf{p}^{\mathrm{fl}}(t)}{\partial t}+\mathbf{E}^{\mathrm{fl}}(\mathbf{r}, t) \cdot \frac{\partial \mathbf{p}^{\mathrm{ind}}(t)}{\partial t}\right\rangle \\
& =P_{p}^{\mathrm{rad}}+P_{E}^{\mathrm{rad}} .
\end{aligned}
$$

The first term on the right-hand side of Eq. (19) accounts for the power radiated by the particle due to fluctuations in its polarization, while the second one describes the power dissipated in the particle due to field fluctuations. The first term can be rewritten as

$$
\begin{aligned}
P_{p}^{\mathrm{rad}} & =-\left\langle\mathbf{E}^{\mathrm{ind}}(\mathbf{r}, t) \cdot \frac{\partial \mathbf{p}^{\mathrm{fl}}(t)}{\partial t}\right\rangle \\
& =\int_{-\infty}^{\infty} \frac{d \omega d \omega^{\prime}}{(2 \pi)^{2}} e^{-i\left(\omega+\omega^{\prime}\right) t}\left(i \omega^{\prime}\right) \mathbf{E}^{\mathrm{ind}}\left(\mathbf{r}, \omega^{\prime}\right) \cdot \mathbf{p}^{\mathrm{fl}}\left(\omega^{\prime}\right) .
\end{aligned}
$$

Now, we express the induced field in terms of the fluctuating dipole using Eq. (2). Then, the dipole can be written in the rotating frame with the help of Eq. (9) and we can apply the FDT [Eq. (4)] to obtain

$$
\begin{aligned}
P_{p}^{\mathrm{rad}}= & -\frac{i \hbar}{2 \pi} \int_{-\infty}^{\infty} d \omega \omega\left\{\left[G_{x x}(\mathbf{r}, \mathbf{r}, \omega)+G_{y y}(\mathbf{r}, \mathbf{r}, \omega)\right]\right. \\
& \times\left[\operatorname{Im}\left\{\alpha_{\perp}\left(\omega_{+}\right)\right\}\left(n_{1}\left(\omega_{+}\right)+\frac{1}{2}\right)\right. \\
& \left.+\operatorname{Im}\left\{\alpha_{\perp}\left(\omega_{-}\right)\right\}\left(n_{1}\left(\omega_{-}\right)+\frac{1}{2}\right)\right] \\
& \left.+2 G_{z z}(\mathbf{r}, \mathbf{r}, \omega)\left[\operatorname{Im}\left\{\alpha_{\|}(\omega)\right\}\left(n_{1}(\omega)+\frac{1}{2}\right)\right]\right\} .
\end{aligned}
$$

Notice that we have already integrated over $\omega^{\prime}$ and, although the $z$ component does not contribute to the torque, it does radiate power.

Finally, using the symmetry properties of the Green tensor, we can write

$$
\begin{aligned}
P_{p}^{\mathrm{rad}}= & \frac{4 \hbar}{3 \pi c^{3}} \int_{0}^{\infty} d \omega \omega^{4}\left[\operatorname{Im}\left\{\alpha_{\perp}\left(\omega_{+}\right)\right\}\left(n_{1}\left(\omega_{+}\right)+\frac{1}{2}\right)\right. \\
& \left.+\operatorname{Im}\left\{\alpha_{\perp}\left(\omega_{-}\right)\right\}\left(n_{1}\left(\omega_{-}\right)+\frac{1}{2}\right)\right] \\
& +\frac{4 \hbar}{3 \pi c^{3}} \int_{0}^{\infty} d \omega \omega^{4} \operatorname{Im}\left\{\alpha_{\|}(\omega)\right\}\left(n_{1}(\omega)+\frac{1}{2}\right),
\end{aligned}
$$

where we have used Eq. (11). This result represents the power radiated by the rotating particle due to the dipole fluctuations.

The remaining term of Eq. (19) represents the radiation dissipated in the particle due to the fluctuations of the external field, which can be written as

$$
\begin{aligned}
P_{E}^{\mathrm{rad}} & =-\left\langle\mathbf{E}^{\mathrm{fl}}(\mathbf{r}, t) \cdot \frac{\partial \mathbf{p}^{\mathrm{ind}}(t)}{\partial t}\right\rangle \\
& =\int_{-\infty}^{\infty} \frac{d \omega d \omega^{\prime}}{(2 \pi)^{2}} e^{-i\left(\omega+\omega^{\prime}\right) t}\left(i \omega^{\prime}\right) \mathbf{E}^{\mathrm{fl}}(\mathbf{r}, \omega) \cdot \mathbf{p}^{\mathrm{ind}}\left(\omega^{\prime}\right) .
\end{aligned}
$$

Using the effective polarizability [Eq. (14)], the FDT [Eq. (5)], and Eq. (11), we find

$$
\begin{aligned}
P_{E}^{\mathrm{rad}}= & -\frac{2 i \hbar}{3 \pi c^{3}} \int_{-\infty}^{\infty} d \omega \omega^{4}\left[\alpha_{\perp}^{*}\left(\omega_{+}\right)+\alpha_{\perp}^{*}\left(\omega_{-}\right)+\alpha_{\|}^{*}(\omega)\right] \\
& \times\left(n_{0}(\omega)+\frac{1}{2}\right),
\end{aligned}
$$

where we have already integrated over $\omega^{\prime}$. Using again the causality of the polarizability and noticing that the integrand is an odd function of $\omega$, Eq. (21) becomes

$$
\begin{aligned}
P_{E}^{\mathrm{rad}}= & -\frac{4 \hbar}{3 \pi c^{3}} \int_{0}^{\infty} d \omega \omega^{4}\left[\operatorname{Im}\left\{\alpha_{\perp}\left(\omega_{+}\right)\right\}+\operatorname{Im}\left\{\alpha_{\perp}\left(\omega_{-}\right)\right\}\right. \\
& \left.+\operatorname{Im}\left\{\alpha_{\|}(\omega)\right\}\right]\left(n_{0}(\omega)+\frac{1}{2}\right) .
\end{aligned}
$$

Finally, the net power radiated by the particle, which results from the difference between the power radiated by dipole fluctuations [Eq. (20)] and the power dissipated by the fluctuating field [Eq. (22)], can be written as

$$
\begin{aligned}
P^{\mathrm{rad}}= & \frac{4 \hbar}{3 \pi c^{3}} \int_{0}^{\infty} d \omega \omega^{4} \operatorname{Im}\left\{\alpha_{\perp}\left(\omega_{-}\right)\right\}\left[n_{1}\left(\omega_{-}\right)-n_{0}(\omega)\right] \\
& +\frac{4 \hbar}{3 \pi c^{3}} \int_{0}^{\infty} d \omega \omega^{4} \operatorname{Im}\left\{\alpha_{\perp}\left(\omega_{+}\right)\right\}\left[n_{1}\left(\omega_{+}\right)-n_{0}(\omega)\right] \\
& +\frac{4 \hbar}{3 \pi c^{3}} \int_{0}^{\infty} d \omega \omega^{4} \operatorname{Im}\left\{\alpha_{\|}(\omega)\right\}\left[n_{1}(\omega)-n_{0}(\omega)\right] .
\end{aligned}
$$

A more compact expression is obtained by playing with the integration limits:

$$
\begin{aligned}
P^{\mathrm{rad}}= & \frac{4 \hbar}{3 \pi c^{3}} \int_{-\infty}^{\infty} d \omega \omega^{4} \operatorname{Im}\left\{\alpha_{\perp}\left(\omega_{-}\right)\right\}\left[n_{1}\left(\omega_{-}\right)-n_{0}(\omega)\right] \\
& +\frac{2 \hbar}{3 \pi c^{3}} \int_{-\infty}^{\infty} d \omega \omega^{4} \operatorname{Im}\left\{\alpha_{\|}(\omega)\right\}\left[n_{1}(\omega)-n_{0}(\omega)\right] .
\end{aligned}
$$

It should be noted that even if the particle is not rotating there is a net radiated power when the particle and vacuum temperatures are different.

As in the case of the torque, we can generalize this result to anisotropic particles, and we also find that Eq. (24) is still valid if $\alpha_{\perp}(\omega)$ is understood as the average of the polarizabilities along the $x$ and $y$ axes.

\section{QUANTUM-MECHANICAL APPROACH}

One can question the suitability of the FDT to deal with a system that is out of dynamical equilibrium, such as our rotating particle when it is experiencing friction. We clarify this point and achieve deeper understanding of vacuum and 
thermal friction by solving the problem within the framework of quantum electrodynamics. As we show next, this procedure leads to analytical expressions for the torque and the radiated power that are identical with the ones obtained from the FDT.

The rotation of the particle around the $z$ axis can be described by the Hamiltonian

$$
\hat{H}_{\mathrm{rot}}=\frac{L_{z}^{2}}{2 I}=-\frac{\hbar^{2}}{2 I} \frac{\partial^{2}}{\partial \varphi^{2}},
$$

where $I$ is the particle moment of inertia. Accordingly, the rotational part of the eigenfunction can be written

$$
\Psi_{R}(\varphi, t)=\frac{1}{\sqrt{2 \pi}} e^{i m \varphi} e^{\left(-i \hbar m^{2} / 2 I\right) t},
$$

which describes a rotation of frequency $\Omega=\hbar m / I$, as obtained from the condition of the stationary phase [19]. This wave function satisfies the equation

$$
\hat{H}_{\text {rot }} \Psi_{\text {rot }}(\varphi)=E_{\text {rot }} \Psi_{\text {rot }}(\varphi),
$$

where $E_{\mathrm{rot}}=\hbar^{2} m^{2} / 2 I$ is the rotational energy.

The complete system (particle plus field) can be adequately described by states $\left|k_{j} m l_{i}\right\rangle$, where $k_{j}$ is the number of particle bosonic excitations of energy $\varepsilon_{j}$ in the particle internal state (e.g., phonons or plasmons), $m$ is the rotational azimuthal number, and $l_{i}$ describes the electromagnetic field state through the number of photons in mode $i$. The energy of this state is $k_{j} \varepsilon_{j}+\hbar^{2} m^{2} / 2 I+l_{i} \hbar \omega_{i}$.

Using Fermi's "golden rule" [20] to account for the particleradiation coupling to first order, the transition probabilities between states like the one defined above can be written

$$
\begin{aligned}
P_{\left|k_{j}^{\prime} m^{\prime} l_{i}^{\prime}\right\rangle \leftarrow\left|k_{j} m l_{i}\right\rangle}= & \frac{2 \pi}{\hbar}\left|\left\langle k_{j}^{\prime} m^{\prime} l_{i}^{\prime}\left|\hat{H}_{I}\right| k_{j} m l_{i}\right\rangle\right|^{2} \\
& \times \delta\left(\Delta k_{j} \varepsilon_{j}+\hbar^{2} \frac{m^{2}-m^{2}}{2 I}+\Delta l_{i} \hbar \omega_{i}\right),
\end{aligned}
$$

where $\Delta k_{j}=k_{j}^{\prime}-k_{j}$ and $\Delta l_{i}=l_{i}^{\prime}-l_{i}$. Here, $\hat{H}_{I}$ is the Hamiltonian that describes the interaction between the particle and the radiation field. For small particles, $\hat{H}_{I}$ is well described by the dipolar interaction Hamiltonian

$$
\hat{H}_{I}=-\hat{\mathbf{p}} \cdot \hat{\mathbf{E}}(\mathbf{r}) .
$$

For bosonic excitations (e.g., phonons or plasmons) the dipole operator $\hat{\mathbf{p}}$ can be written in terms of particle bosonic creation and annihilation operators $\hat{b}^{\dagger}$ and $b$ as [21]

$$
\hat{\mathbf{p}}=\sum_{j} \mathbf{p}_{j}\left[\hat{b}_{j}+\hat{b}_{j}^{\dagger}\right]
$$

where $\mathbf{p}_{j}$ is the dipole moment associated to the bosonic mode $j$ [22]. Likewise, the electric-field operator can be expressed in terms of photon creation and annihilation operators $\hat{a}^{\dagger}$ and $a$ as

$$
\hat{\mathbf{E}}(\mathbf{r})=i \sum_{\mathbf{k} \sigma} \sqrt{\frac{2 \pi \hbar \omega_{\mathbf{k}}}{V}} \mathbf{e}_{\mathbf{k} \sigma}\left[\hat{a}_{\mathbf{k} \sigma} e^{i \mathbf{k} \cdot \mathbf{r}}-\hat{a}_{\mathbf{k} \sigma}^{\dagger} e^{-i \mathbf{k} \cdot \mathbf{r}}\right],
$$

where $V$ is the quantization volume, $\mathbf{e}_{\mathbf{k} \sigma}$ is the mode polarization vector, and the sum runs over photon wave vectors $\mathbf{k}$ and polarizations $\sigma$ [i.e., the photon index $i$ in Eq. (26) contains the photon wave vector and the polarization].
In the dipolar approximation, we can take $e^{i \mathbf{k} \cdot \mathbf{r}} \approx 1$, which, together with Eq. (25), allows us to write the relation

$$
\begin{aligned}
\left\langle k_{j}^{\prime} m^{\prime} l_{i}^{\prime}\left|H_{I}\right| k_{j} m l_{i}\right\rangle= & -i \sqrt{\frac{2 \pi \hbar \omega_{i}}{V}} N \\
& \times \int\left(\mathbf{p}_{j} \cdot \mathbf{e}_{i}\right) \frac{e^{i\left(m-m^{\prime}\right) \varphi}}{2 \pi} d \varphi
\end{aligned}
$$

for the transition matrix elements. Here, $N$ is the matrix element corresponding to the bosonic and photonic degrees of freedom, which produce four possible transitions depending on the change of the bosonic $\left(\Delta k_{j}= \pm 1\right)$ and photonic $\left(\Delta l_{i}= \pm 1\right)$ numbers. More precisely,

$$
\begin{gathered}
\left\langle k_{j}-1 l_{i}-1\left|\hat{b}_{j} \hat{a}_{i}\right| k_{j} l_{i}\right\rangle \rightarrow N=\sqrt{k_{j} l_{i}}, \\
\left\langle k_{j}+1 l_{i}+1\left|\hat{b}_{j}^{\dagger} \hat{a}_{i}^{\dagger}\right| k_{j} l_{i}\right\rangle \rightarrow N=-\sqrt{\left(k_{j}+1\right)\left(l_{i}+1\right)}, \\
\left\langle k_{j}+1 l_{i}-1\left|\hat{b}_{j}^{\dagger} \hat{a}_{i}\right| k_{j} l_{i}\right\rangle \rightarrow N=\sqrt{\left(k_{j}+1\right) l_{i}}, \\
\left\langle k_{j}-1 l_{i}+1\left|\hat{b}_{j} \hat{a}_{i}^{\dagger}\right| k_{j} l_{i}\right\rangle \rightarrow N=-\sqrt{k_{j}\left(l_{i}+1\right)},
\end{gathered}
$$

Now, the integration over $\varphi$ in Eq. (28) requires expressing the dipole moment in the particle frame using the relations

$$
\begin{gathered}
p_{x}=p_{x}^{\prime} \cos \varphi-p_{y}^{\prime} \sin \varphi, \\
p_{y}=p_{x}^{\prime} \sin \varphi+p_{y}^{\prime} \cos \varphi, \\
p_{z}=p_{z}^{\prime},
\end{gathered}
$$

where the primed (nonprimed) quantities refer to the rotating (lab) frame. From here we have

$$
\begin{aligned}
\left\langle k_{j}^{\prime} m^{\prime} l_{i}^{\prime}\left|H_{I}\right| k_{j} m l_{i}\right\rangle= & -i \sqrt{\frac{2 \pi \hbar \omega_{i}}{V}} N \frac{1}{2} \\
& \times\left\{\left[p_{j, x}\left(\delta_{m, m^{\prime}+1}+\delta_{m, m^{\prime}-1}\right)\right.\right. \\
& \left.+i p_{j, y}\left(\delta_{m, m^{\prime}+1}-\delta_{m, m^{\prime}-1}\right)\right] e_{i, x} \\
& +\left[-i p_{j, x}\left(\delta_{m, m^{\prime}+1}-\delta_{m, m^{\prime}-1}\right)\right. \\
& \left.+p_{j, y}\left(\delta_{m, m^{\prime}+1}+\delta_{m, m^{\prime}-1}\right)\right] e_{i, y} \\
& \left.+2 p_{j, z} \delta_{m, m^{\prime}} e_{i, z}\right\}
\end{aligned}
$$

where $e_{i, j}$ are the Cartesian components of $\mathbf{e}_{i}$. The Kronecker delta functions appear as a result of the $\varphi$ integration [see Eqs. (28) and (33)]. From these equations we conclude that there is no contribution to the torque coming from the dipole component along the rotation axis $z$, because it conserves the rotation number $m$.

\section{A. Torque}

Each of the four possible transitions discussed in Eqs. (29)(32) contributes to the torque through the rate of change in the rotational energy. More precisely,

$$
\begin{aligned}
M & =\frac{1}{\Omega} \frac{d E_{\text {rot }}}{d t} \\
& =-\sum_{j, m^{\prime}, i} \frac{ \pm \varepsilon_{j} \pm \hbar \omega_{i}}{\Omega} P_{\left|k_{j} \pm 1 m^{\prime} l_{i} \pm 1\right\rangle \leftarrow\left|k_{j} m l_{i}\right\rangle},
\end{aligned}
$$

where the signs depend on the particular transition, according to the energy conservation imposed by the $\delta$-function of 
Eq. (26), and we are summing over all possible final states. Replacing the sum over the photonic states by an integral, $\sum_{i} \rightarrow \int d S \sum_{\sigma}\left[V /(2 \pi)^{3}\right]\left(1 / c^{3}\right) \int_{0}^{\infty} \omega^{2} d \omega$, and using Eq. (34), we find

$$
\begin{aligned}
M= & \frac{-2 \hbar}{3 \Omega c^{3}} \sum_{j, m^{\prime}} \int_{0}^{\infty} d \omega N^{2} \omega^{3}\left( \pm \varepsilon_{j} \pm \hbar \omega\right) \\
& \times\left\{\left|p_{j, x}+i p_{j, y}\right|^{2} \delta_{m^{\prime}, m-1}+\left|p_{j, x}-i p_{j, y}\right|^{2} \delta_{m^{\prime}, m+1}\right\} \\
& \times \delta\left( \pm \varepsilon_{j} \pm \hbar \omega+\hbar^{2} \frac{m^{\prime 2}-m^{2}}{2 I}\right)
\end{aligned}
$$

The factors $\delta_{m^{\prime}, m \pm 1}$ allow us to write

$$
\hbar^{2} \frac{\left(m^{\prime 2}-m^{2}\right)}{2 I}=\hbar^{2} \frac{(m \pm 1)^{2}-m^{2}}{2 I} \approx \pm \hbar \Omega
$$

(with $\Omega=\hbar m / I$ ), where we use the nonrecoil approximation $(|m| \gg 1)$. Using this expression and defining $p_{j}^{2}=\left|p_{j, x} \pm i p_{j, y}\right|^{2}$, we can rewrite Eq. (35) as

$$
\begin{aligned}
M= & -\frac{4 \hbar}{3 c^{3}} \sum_{j} \int_{0}^{\infty} d \omega N^{2} \omega^{3} p_{j}^{2} \\
& \times\left[\delta\left( \pm \varepsilon_{j} \pm \hbar \omega-\hbar \Omega\right)-\delta\left( \pm \varepsilon_{j} \pm \hbar \omega+\hbar \Omega\right)\right],
\end{aligned}
$$

where a factor of 2 has been included to account for mode degeneracy in the sum over $j$ [22], so that $j$ runs now over dipole excitations along either $x$ or $y$ in the axially symmetric particle. Finally, the total torque is the sum of the contributions from the four transitions described in Eqs. (29)-(32).

At this point, we assume that both the radiation field and the particle are in thermodynamic equilibrium at temperatures $T_{0}$ and $T_{1}$, respectively. Then, we have to average over the number of bosons $k_{j}$ and photons $l_{i}$ in the system, which appear in the factor $N$. Using the Bose-Einstein statistics,

$$
\left\langle k_{j}\right\rangle=\frac{1}{e^{\varepsilon_{j} / k_{B} T_{1}}-1}=n_{1}\left(\varepsilon_{j}\right)
$$

and

$$
\left\langle l_{i}\right\rangle=\frac{1}{e^{\hbar \omega_{i} / k_{B} T_{0}}-1}=n_{0}\left(\omega_{i}\right),
$$

the averaged torque reduces to

$$
\begin{aligned}
M= & \frac{4 \hbar}{3 c^{3}} \sum_{j} \int_{0}^{\infty} d \omega \omega^{3} p_{j}^{2} \\
& \times\left\{\left[\delta\left(\varepsilon_{j}-\hbar \omega_{+}\right)-\delta\left(\varepsilon_{j}+\hbar \omega_{+}\right)\right]\left[n_{1}\left(\omega_{+}\right)-n_{0}(\omega)\right]\right. \\
& -\left[\delta\left(\varepsilon_{j}-\hbar \omega_{-}\right)-\delta\left(\varepsilon_{j}+\hbar \omega_{-}\right)\right] \\
& \left.\times\left[n_{1}\left(\omega_{-}\right)-n_{0}(\omega)\right]\right\}
\end{aligned}
$$

\section{B. Net radiated power}

The net radiated power can be calculated in a way similar to the torque. Once again, there are four separate contributions associated to the transitions of Eqs. (29)-(32). Two of them involve the emission of a photon (with $\Delta l_{i}=1$ and $\Delta k_{j}=$ \pm 1 ), thus producing a positive contribution, whereas the other two describe photon absorption $\left(\Delta l_{i}=-1\right.$ and $\left.\Delta k_{j}= \pm 1\right)$, giving rise to a negative contribution. The net radiated power can then be written

$$
\begin{aligned}
P^{\mathrm{rad}}= & \sum_{j, m^{\prime}, i} \hbar \omega_{i}\left[P_{\left|k_{j}+1 m^{\prime} l_{i}+1\right\rangle \leftarrow\left|k_{j} m l_{i}\right\rangle}+P_{\left|k_{j}-1 m^{\prime} l_{i}+1\right\rangle \leftarrow\left|k_{j} m l_{i}\right\rangle}\right. \\
& \left.-P_{\left|k_{j}+1 m^{\prime} l_{i}-1\right\rangle \leftarrow\left|k_{j} m l_{i}\right\rangle}-P_{\left|k_{j}-1 m^{\prime} l_{i}-1\right\rangle \leftarrow\left|k_{j} m l_{i}\right\rangle}\right] . \quad \text { (40) }
\end{aligned}
$$

Following the same steps as in Eqs. (35)-(39), we find

$$
\begin{aligned}
P^{\mathrm{rad}}= & \frac{4 \hbar}{3 c^{3}} \sum_{j} \int_{0}^{\infty} d \omega \omega^{4} p_{j}^{2} \\
& \times\left\{\left[n_{1}\left(\omega_{+}\right)-n_{0}(\omega)\right]\left[\delta\left(\varepsilon_{j}-\hbar \omega_{+}\right)-\delta\left(\varepsilon_{j}+\hbar \omega_{+}\right)\right]\right. \\
& +\left[n_{1}\left(\omega_{-}\right)-n_{0}(\omega)\right]\left[\delta\left(\varepsilon_{j}-\hbar \omega_{-}\right)-\delta\left(\varepsilon_{j}+\hbar \omega_{-}\right)\right] \\
& \left.+\left[n_{1}(\omega)-n_{0}(\omega)\right]\left[\delta\left(\varepsilon_{j}-\hbar \omega\right)-\delta\left(\varepsilon_{j}+\hbar \omega\right)\right]\right\} .
\end{aligned}
$$

It should be noted that, unlike the torque, the radiated power contains a contribution from the dipole component parallel to the rotation axis.

\section{EQUIVALENCE OF THE FLUCTUATION-DISSIPATION THEOREM AND THE QUANTUM-MECHANICAL APPROACHES}

In order to compare the expressions for the torque and the net radiated power obtained within the two different approaches, we have to introduce the particle polarizability $\alpha$ in the QM formalism. From the customary definition [23]

$$
\alpha(\omega)=\sum_{j} p_{j}^{2}\left[\frac{1}{\varepsilon_{j}-\hbar \omega-i \gamma}+\frac{1}{\varepsilon_{j}+\hbar \omega+i \gamma}\right],
$$

and using the identity

$$
\mp \delta(x)=\frac{1}{\pi} \operatorname{Im}\left\{\frac{1}{x \pm i \gamma}\right\},
$$

where $\gamma \rightarrow 0^{+}$, we can write the imaginary part of the polarizability as

$$
\operatorname{Im}\{\alpha(\omega)\}=\pi \sum_{j} p_{j}^{2}\left[\delta\left(\varepsilon_{j}-\hbar \omega\right)-\delta\left(\varepsilon_{j}+\hbar \omega\right)\right] .
$$

This allows us to directly recast the torque and the radiated power given by Eqs. (39) and (41) into exactly the same form as in Eqs. (17) and (23), respectively. The only difference is that we have focused on isotropic particles in the QM approach, although the generalization to anisotropic particles can be easily done through the dipole moment of the bosonic excitations $\mathbf{p}_{j}$ [see Eq. (27)].

Therefore we find that the FDT and the QM approaches lead to exactly the same results. An additional conclusion from the QM derivation is that isotropic particles with no allowed internal excitations (i.e., nonabsorbing particles) cannot experience any torque, within the limits of our model.

\section{QUANTUM-MECHANICAL APPROACH FOR FERMIONIC SYSTEMS}

In the QM derivation developed in Sec. III, we have considered that the response of the particle to the external field is mediated by bosonic excitations, such as phonons or plasmons. Although such a model describes most situations encountered in practice, we find it very interesting to investigate the vacuum and thermal friction acting on rotating systems 
whose electromagnetic response is governed by fermionic excitations, such as, for instance, a two-level molecule (e.g., a conjugated polymer with a strong dipole-active excitation). In such systems the dipolar operator is given by

$$
\hat{\mathbf{p}}=\sum_{j} \mathbf{p}_{j}\left[\left|g_{j}\right\rangle\left\langle e_{j}|+| e_{j}\right\rangle\left\langle g_{j}\right|\right],
$$

where $\left|g_{j}\right\rangle$ and $\left|e_{j}\right\rangle$ are pairs of possible ground and excited internal states, and $\mathbf{p}_{j}$ is the dipole moment of the fermionic excitation $j$.

Using this dipolar operator instead of Eq. (27), we can calculate the torque and the radiated power following the same procedure as in Sec. III. Once again, there are four possible transitions resulting from the combination of a change in the internal state (ground $\leftrightarrow$ excited) and the absorption or emission of one photon. The torque associated to each of these transitions is given by Eq. (37), where $N^{2}=l_{i}+1$ if a photon is emitted and $N^{2}=l_{i}$ if it is absorbed.

Assuming the vacuum field in thermodynamic equilibrium at temperature $T_{0}$, we take the average of $l_{i}$ using the Bose-Einstein distribution function [Eq. (38)]. Likewise, if the particle is in thermodynamic equilibrium at temperature $T_{1}$, we have to weigh each possible transition with the probability of being initially at the ground state or at the excited state. Such probabilities are given by $1 /\left(1+e^{\varepsilon_{j} / k_{B} T_{1}}\right)$ for the excited state and $e^{\varepsilon_{j} / k_{B} T_{1}} /\left(1+e^{\varepsilon_{j} / k_{B} T_{1}}\right)$ for the ground state, where $\varepsilon_{j}$ is the energy that separates both states. From these considerations, the frictional torque becomes

$$
\begin{aligned}
M= & \frac{-4 \hbar}{3 c^{3}} \sum_{j} \int_{-\infty}^{\infty} d \omega \omega^{3} p_{j}^{2}\left\{\delta\left(\varepsilon_{j}-\hbar \omega_{-}\right)+\delta\left(\varepsilon_{j}+\hbar \omega_{-}\right)\right\} \\
& \times\left[n_{1 F}\left(\omega_{-}\right)+2 n_{1 F}\left(\omega_{-}\right) n_{0}(\omega)-n_{0}(\omega)\right]
\end{aligned}
$$

and similarly, the net radiated power reads

$$
\begin{aligned}
P^{\mathrm{rad}}= & \frac{4 \hbar}{3 c^{3}} \sum_{j} \int_{-\infty}^{\infty} d \omega \omega^{4} p_{j}^{2}\left\{\left[\delta\left(\varepsilon_{j}-\hbar \omega_{-}\right)+\delta\left(\varepsilon_{j}+\hbar \omega_{-}\right)\right]\right. \\
& \times\left[n_{1 F}\left(\omega_{-}\right)+2 n_{0}(\omega) n_{1 F}\left(\omega_{-}\right)-n_{0}(\omega)\right] \\
& +\frac{1}{2}\left[\delta\left(\varepsilon_{j}-\hbar \omega\right)+\delta\left(\varepsilon_{j}+\hbar \omega\right)\right] \\
& \left.\times\left[n_{1 F}(\omega)+2 n_{0}(\omega) n_{1 F}(\omega)-n_{0}(\omega)\right]\right\},
\end{aligned}
$$

where $n_{0}(\omega)$ is the Bose-Einstein distribution function given in Eq. (6) and $n_{1 F}$ is the Fermi-Dirac distribution function

$$
n_{1 F}(\omega)=\frac{1}{e^{\hbar \omega / k_{B} T_{1}}+1} .
$$

In contrast to the bosonic case [Eqs. (39) and (41)], the delta functions in these expressions cannot be written in terms of the imaginary part of the polarizability [Eq. (42)]. However, at zero temperature $T_{0}=T_{1}=0$, these formulas agree with the bosonic expressions. The particle temperature for fermions must be understood in the statistical sense, that is, either for a particle containing many degenerate excitations with identical dipoles or as a time average assuming negligible stopping over many excitation and de-excitation cycles. More precisely, the particle equilibrium temperature is determined by the condition $M \Omega+P^{\mathrm{rad}}=0$. In particular, with the particle hosting only one mode $j=0$ and the vacuum at $T_{0}=0$, we obtain $k_{B} T_{1}=\epsilon_{0} / \ln \left\{\left[\left(\hbar \Omega+\epsilon_{0}\right)^{3}+\epsilon_{0}^{3}\right] /\left(\hbar \Omega-\epsilon_{0}\right)^{3}\right\}$ for $\hbar \Omega>\epsilon_{0}$, and $T_{1}=0$ otherwise. This result concides with the bosonic case under these conditions and it predicts a linear dependence $k_{B} T_{1} \approx \hbar \Omega / 6$ for $\hbar \Omega \gg \epsilon_{0}$.

\section{CONTRIBUTION OF MAGNETIC POLARIZATION}

So far we have only considered the contribution to the torque and the radiated power coming from electric polarization of the particle, but the contribution resulting from magnetic polarization can also play a role. Intuitively, one can be inclined to ignore such contribution for small particles, which have small magnetic polarizability, but we show below that it can actually be dominant in metal particles of large conductivity.

Figure 3 shows the imaginary part of the electric (black curves) and magnetic (red curves) polarizabilities of graphite (a) and gold (b) nanoparticles, with radii of $10 \mathrm{~nm}$ (broken curves) and $100 \mathrm{~nm}$ (solid curves). They have been obtained from the dipolar Mie scattering coefficients $t_{1}^{E}$ and $t_{1}^{M}$ [24] using the relations $\alpha_{\text {elec }}=\left(3 c^{3} / 2 \omega^{3}\right) t_{1}^{E}$ and $\alpha_{\text {mag }}=$ $\left(3 c^{3} / 2 \omega^{3}\right) t_{1}^{M}$. The dielectric functions of graphite and gold have been taken from [25] and [26], respectively. The former includes the effect of the finite size of the particle. From this plot one can clearly observe that the imaginary part of the electric polarizability governs the response of the graphite nanoparticles almost over the whole range of frequencies under consideration. However, the situation is completely reversed in gold nanoparticles, for which the magnetic polarization dominates the infrared spectral range [see Fig. 3(b)].

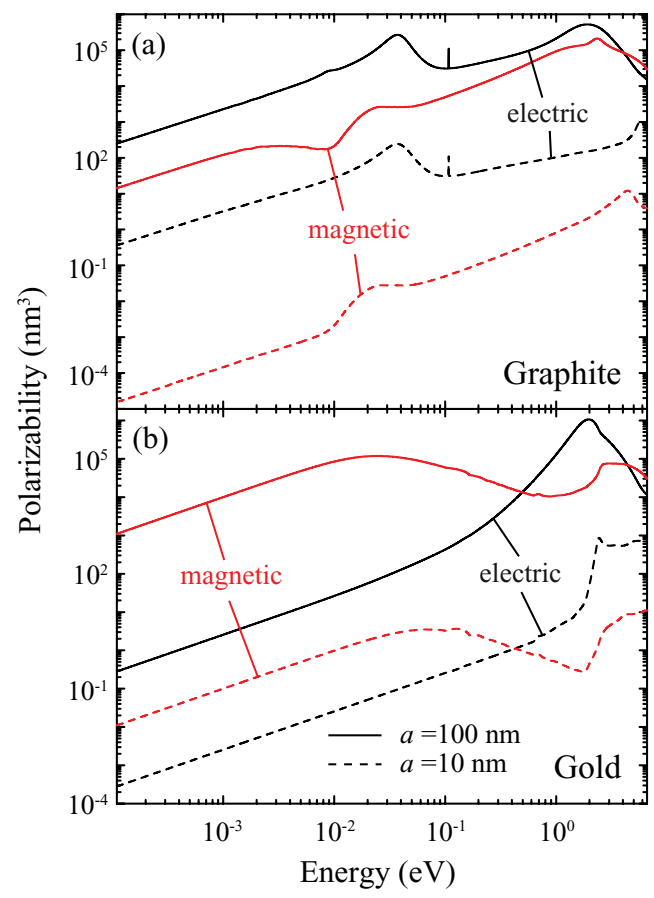

FIG. 3. (Color online) Imaginary part of the electric (black curves) and magnetic (red curves) polarizabilities of graphite (a) and gold (b) nanoparticles of $10 \mathrm{~nm}$ (broken curves) and $100 \mathrm{~nm}$ (solid curves) radius, obtained from the dipolar Mie coefficient. The dielectric functions of graphite and gold have been taken from [25] and [26], respectively. 
It is possible to derive analytical expressions for the magnetic and electric polarizabilities in the low-frequency limit, well below the interband transitions. The dielectric function of metals can then be accurately described by the Drude model in terms of the dc conductivity $\sigma_{0}$ :

$$
\epsilon \approx i \frac{4 \pi \sigma_{0}}{\omega} .
$$

(Incidentally, the real part of $\epsilon$ is negligible compared to the imaginary part in the $\omega \rightarrow 0$ limit and its actual value is irrelevant in the analysis that follows.) Using the small-radius expansion of the Mie scattering coefficients $(a / \lambda \ll 1)$, we find

$$
\alpha_{\mathrm{elec}} \approx a^{3} \frac{\epsilon-1}{\epsilon+2}
$$

and

$$
\alpha_{\mathrm{mag}} \approx \frac{2 \pi^{2}}{15} a^{3}\left(\frac{a}{\lambda}\right)^{2}(\epsilon-1) .
$$

Upon insertion of Eq. (45) into these expressions, the ratio of the imaginary parts of these polarizabilities reduces to

$$
\frac{\operatorname{Im}\left\{\alpha_{\text {mag }}\right\}}{\operatorname{Im}\left\{\alpha_{\text {elec }}\right\}} \approx \frac{8 \pi^{2}}{45}\left(\frac{a \sigma_{0}}{c}\right)^{2} .
$$

Hence the magnetic polarization becomes important at low frequencies in particles of large conductivity. This is precisely what we observe in Fig. 3(b) for gold nanoparticles $\left(\sigma_{0} \approx\right.$ $\left.1.6 \times 10^{7} \Omega^{-1} \mathrm{~m}^{-1}\right)$, in contrast to graphite nanoparticles $\left[\sigma_{0}=2.3 \times 10^{4}\left(2.0 \times 10^{5}\right) \Omega^{-1} \mathrm{~m}^{-1}\right.$ for $\left.a=10(100) \mathrm{nm}\right]$.

\section{A. Magnetic contribution to the torque and the net radiated power}

The magnetic contribution to the torque can be obtained from the expression

$$
M_{\mathrm{mag}}=\langle\mathbf{m}(t) \times \mathbf{H}(\mathbf{r}, t)\rangle \cdot \mathbf{z} .
$$

In a similar way, the net radiated power due to the magnetic polarization reads

$$
P_{\mathrm{mag}}^{\mathrm{rad}}=-\left\langle\mathbf{H}(\mathbf{r}, t) \cdot \frac{\partial \mathbf{m}(t)}{\partial t}\right\rangle .
$$

From these expressions, taking advantage of the symmetry of the Maxwell equations [27], we can follow exactly the same steps as in the derivation shown in Sec. II, just replacing the electric dipole and fields by their magnetic counterparts. Therefore the final expressions for the torque and the net radiated power resulting from the contribution of the magnetic polarization are exactly the same as the ones given in Eqs. (18) and (24), respectively, where $\alpha$ must now be replaced by the magnetic polarizability. There are not cross terms between electric and magnetic polarizabilities.

Figure 4 shows the electric (black curves) and magnetic (red curves) contributions to the torque acting on graphite (a) and gold (b) nanoparticles as a function of temperature (we assume that $T_{1}=T_{0}$ ). Two different values of the particle radius have been considered: $10 \mathrm{~nm}$ (broken curves) and $100 \mathrm{~nm}$ (solid curves). In addition, the particles are rotating with a frequency of $\Omega=10^{4} \mathrm{~Hz}$ (the torque

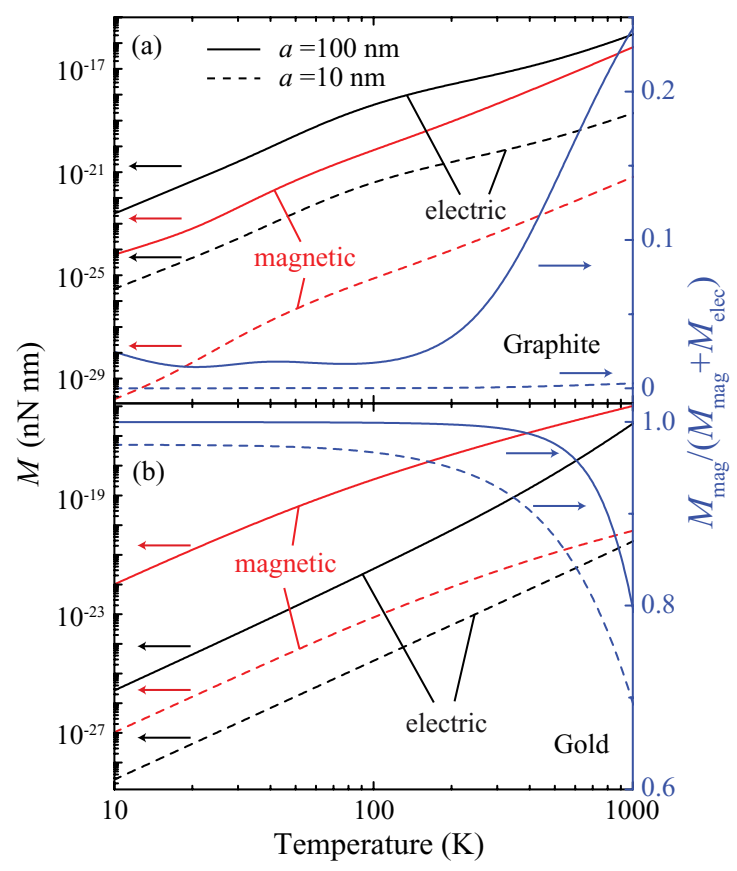

FIG. 4. (Color online) Electric (black curves) and magnetic (red curves) contributions to the torque acting on graphite (a) and gold (b) nanoparticles of $10 \mathrm{~nm}$ (broken curves) and $100 \mathrm{~nm}$ (solid curves) radius for different temperatures (we take $T_{1}=T_{0}$ ). The particles are rotating with a frequency of $10^{4} \mathrm{~Hz}$. The blue lines (right scale) show the relative weight of magnetic polarization contributing to the torque.

is proportional to $\Omega$ at this low rotation velocity). Both contributions to the torque grow with temperature. The blue curves in Fig. 4 (right scale) show the relative weight of the contribution to the torque coming from magnetic polarization. Such contribution is negligible in the 10-nm-radius graphite nanoparticle [see Fig. 4(a)], although it becomes noticeable for a radius of $100 \mathrm{~nm}$ at temperatures above $\sim 300 \mathrm{~K}$. The situation is completely reversed in the gold nanoparticles [see Fig. 4(b)], for which the magnetic contribution almost dominates the torque over the whole temperature range, and only for temperatures above $300 \mathrm{~K}$ does the electric contribution take comparatively significant values.

\section{B. Stopping times of interstellar dust}

In the common situation of low rotation frequencies compared to the thermal energy (i.e., $\Omega \ll k T / \hbar$, which implies $\Omega \ll 21 \mathrm{GHz}$ for a temperature of $1 \mathrm{~K}$ ), and assuming the particle to be at the same temperature as the vacuum $\left(T_{1}=T_{0}\right)$, the torque [Eq. (18)] becomes linear in $\Omega$ :

$$
M \approx \frac{4 \hbar}{3 \pi c^{3}} \Omega \int_{-\infty}^{\infty} d \omega \omega^{3} \operatorname{Im}\left\{\alpha_{\perp}(\omega)\right\} \frac{\partial n_{1}(\omega)}{\partial \omega} .
$$

Combining this result and Newton's second law, we can write the time evolution of the rotation frequency as

$$
\Omega(t)=\Omega(0) e^{-t / \tau},
$$




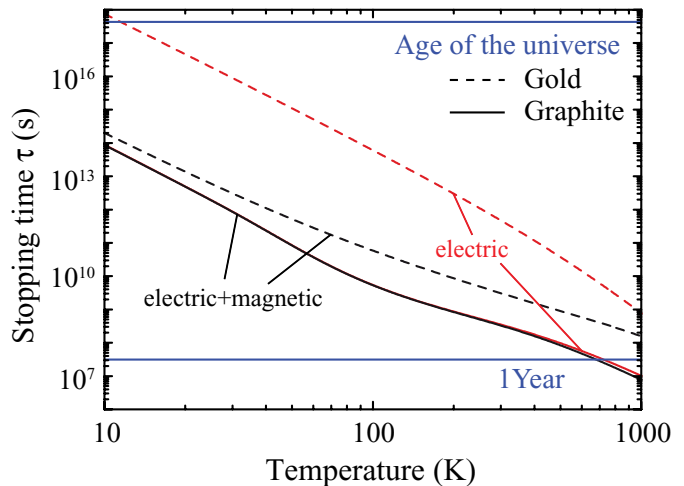

FIG. 5. (Color online) Stopping times of graphite (solid curves) and gold (broken curves) nanoparticles of $100 \mathrm{~nm}$ radius, with (black curves) and without (red curves) inclusion of magnetic polarization. Electric polarization dominates in graphite, so both black and red curves almost coincide in that case. In contrast, electric polarization is negligible in the case of gold.

where

$$
\tau=\frac{3 \pi c^{3} I}{-4 \hbar \int_{-\infty}^{\infty} d \omega \omega^{3} \operatorname{Im}\left\{\alpha_{\perp}(\omega)\right\}\left[\partial n_{1}(\omega) / \partial \omega\right]}
$$

is the characteristic $1 / e$ stopping time and $I$ is the moment of inertia.

Graphite particles are abundant in interstellar dust [28], so they constitute a relevant case to study the stopping time $\tau$. The black solid curve in Fig. 5 shows the variation of this parameter as a function of temperature for a graphite particle of $100 \mathrm{~nm}$ radius. The stopping time ranges from cosmic-scale values at low temperatures to much smaller times at higher temperatures. Figure 5 shows the particle stopping time including both electric and magnetic polarization (black curves) compared to the value obtained by neglecting the magnetic contribution (red curves). As expected from the discussion of Sec. VI A, only the electric component is relevant for graphite nanoparticles. For completeness, we include in Fig. 5 the stopping time of gold nanoparticles (broken curves), for which the magnetic contribution dominates the stopping time nearly over the whole range of temperatures.

We have neglected here the variation of the particle polarizability with temperature, which constitutes a minor correction except at very high temperatures or when phase transitions are involved. Furthermore, we have ignored the effect of centrifugal forces on the particle response, which should be negligible at moderate rotational frequencies. For instance, the centrifugal energy of outer electrons in a 200-nm gold nanoparticle is of the order of the Fermi energy only above $1 \mathrm{THz}$ rotation frequencies.

\section{CONCLUDING REMARKS}

In this paper, we have developed a comprehensive semiclassical model based upon the FDT to describe vacuum and thermal friction acting on rotating particles. We have presented analytical expressions both for the torque exerted on an axially symmetric particle and for the net power that it radiates due to friction. We have also generalized these results to the case of anisotropic particles.

The semiclassical approach of Sec. I is in full agreement with the QM derivation presented in Sec. III, in which the particle and the external field are described using combined states that include the internal state of the particle, which can host a number of bosonic excitations (e.g., phonons or plasmons), the rotational state, and the electromagnetic field in the photon-number representation. The coupling between the mechanical rotation and the vacuum photon field is mediated by internal excitations of the particle. Using Fermi's "golden rule" to evaluate the different possible transitions between those states, we calculate the torque and the net radiated power. The final analytical expressions for these magnitudes are the same as those obtained from the FDT approach.

We have extended the QM model to cope with fermionic systems, such as, for instance, a molecule or a quantum dot with an effective two-level electronic structure. This extension has required a careful derivation involving the particle-dipole operator and the Fermi-Dirac statistics.

The contribution to vacuum and thermal friction coming from magnetic polarization has been shown to be important for highly conductive materials (e.g., gold), and it can actually dominate over its electric counterpart. In contrast, it is almost negligible in less conductive materials such as graphite.

Our results can be relevant to the study of the rotational dynamics of cosmic nanoparticles. In a more fundamental direction, they provide solid theoretical support for a semiclassical treatment of fluctuations using the FDT. These methods can be useful to deal with friction in other situations such as sliding surfaces, a particle moving near a surface, and neutral particles in relative contactless motion. An interesting scenario is presented by Casimir-bound objects, orbiting around each other, so that spin-orbit interaction (i.e., coupling between rotational and translational degrees of freedom) can play a significant role.

\section{ACKNOWLEDGMENTS}

This work was supported by the Spanish Ministerio de Ciencia e Innovación (MAT2007-66050 and Consolider NanoLight.es). A.M. acknowledges financial support from the Spanish Ministerio de Educación through the FPU program.

\section{APPENDIX: DERIVATION OF AN EFFECTIVE POLARIZABILITY FOR A ROTATING PARTICLE}

As indicated in the main text, calculating the contribution of field fluctuations to both the torque and the radiated power requires transforming the external field to the rotating frame, in which it produces an induced dipole via the particle polarizability, along with the corresponding induced field, and finally this field has to be transformed back to the rest frame. It is possible to condense these steps in an effective particle polarizability that contains the effect of the rotational motion.

We start by examining the relation between the laboratoryframe induced dipole (nonprimed variables) and the dipole in the rotating frame (primed variables) given by Eq. (9). Using 
the particle polarizability, we can write

$$
\begin{aligned}
& p_{x}(\omega)= \frac{1}{2}\left[\alpha\left(\omega_{+}\right) E_{x}^{\prime}\left(\mathbf{r}, \omega_{+}\right)+i \alpha\left(\omega_{+}\right) E_{y}^{\prime}\left(\mathbf{r}, \omega_{+}\right)\right. \\
&\left.+\alpha\left(\omega_{-}\right) E_{x}^{\prime}\left(\mathbf{r}, \omega_{-}\right)-i \alpha\left(\omega_{-}\right) E_{y}^{\prime}\left(\mathbf{r}, \omega_{-}\right)\right], \\
& p_{y}(\omega)= \frac{1}{2}\left[-i \alpha\left(\omega_{+}\right) E_{x}^{\prime}\left(\mathbf{r}, \omega_{+}\right)+\alpha\left(\omega_{+}\right) E_{y}^{\prime}\left(\mathbf{r}, \omega_{+}\right)\right. \\
&\left.+i \alpha\left(\omega_{-}\right) E_{x}^{\prime}\left(\mathbf{r}, \omega_{-}\right)+\alpha\left(\omega_{-}\right) E_{y}^{\prime}\left(\mathbf{r}, \omega_{-}\right)\right], \\
& p_{z}(\omega)=\alpha(\omega) E_{z}^{\prime}(\mathbf{r}, \omega),
\end{aligned}
$$

where $E^{\prime}$ is the field in the rotating frame. Now, we insert the field in the rest frame using

$$
\begin{aligned}
E_{x}^{\prime}(\mathbf{r}, \omega)= & \frac{1}{2}\left[E_{x}\left(\mathbf{r}, \omega_{+}\right)-i E_{y}\left(\mathbf{r}, \omega_{+}\right)\right. \\
& \left.+E_{x}\left(\mathbf{r}, \omega_{-}\right)+i E_{y}\left(\mathbf{r}, \omega_{-}\right)\right], \\
E_{y}^{\prime}(\mathbf{r}, \omega)= & \frac{1}{2}\left[i E_{x}\left(\mathbf{r}, \omega_{+}\right)+E_{y}\left(\mathbf{r}, \omega_{+}\right)\right. \\
& \left.-i E_{x}\left(\mathbf{r}, \omega_{-}\right)+E_{y}\left(\mathbf{r}, \omega_{-}\right)\right], \\
& E_{z}^{\prime}(\mathbf{r}, \omega)=E_{z}(\mathbf{r}, \omega) .
\end{aligned}
$$

Finally, introducing these expressions into Eq. (46), we find $\mathbf{p}=\alpha^{\text {eff }} \mathbf{E}$, from where one readily obtains Eq. (14) for the components of the rest-frame polarizability $\alpha^{\mathrm{eff}}$.
[1] E. J. Post, Rev. Mod. Phys. 39, 475 (1967).

[2] I. Bialynicki-Birula and Z. Bialynicka-Birula, Phys. Rev. Lett. 78, 2539 (1997).

[3] M. Michalski, W. Huttner, and H. Schimming, Phys. Rev. Lett. 95, 203005 (2005).

[4] K. Briggs, Aust. J. Phys. 41, 629 (1988).

[5] D. Park, Phys. Rev. 99, 1324 (1955).

[6] F. S. Chute, IEEE Trans. Antennas Propag. 15, 585 (1967).

[7] P. Galajda and P. Ormos, Appl. Phys. Lett. 78, 249 (2001).

[8] S. H. Tao et al., Opt. Express 13, 7726 (2005).

[9] O. Kenneth and S. Nussinov, Phys. Rev. D 65, 085014 (2002).

[10] M. Kardar and R. Golestanian, Rev. Mod. Phys. 71, 1233 (1999).

[11] J. B. Pendry, J. Phys.: Condens. Matter 9, 10301 (1997).

[12] A. I. Volokitin and B. N. J. Persson, Rev. Mod. Phys. 79, 1291 (2007).

[13] T. G. Philbin and U. Leonhardt, New J. Phys. 11, 033035 (2009); G. V. Dedkov and A. A. Kyasov, Surf. Sci. 561, 604 (2010); J. B. Pendry, New J. Phys. 12, 033028 (2010); U. Leonhardt, ibid. 12, 068001 (2010); J. B. Pendry, ibid. 12, 068002 (2010).

[14] A. Manjavacas and F. J. García de Abajo, Phys. Rev. Lett. 105, 113601 (2010).

[15] H. B. Callen and T. A. Welton, Phys. Rev. 83, 34 (1951).

[16] R. Kubo, Rep. Prog. Phys. 29, 255 (1966).
[17] Y. Pomeau, J. Stat. Phys. 121, 1083 (2005); Europhys. Lett. 74, 951 (2006).

[18] These conditions are easily fulfilled in most situations encountered in practice, for instance, a particle of $100 \mathrm{~nm}$ in diameter requires $\Omega \ll 6 \times 10^{3} \mathrm{THz}$ and $T_{j} \ll 4.6 \times 10^{4} \mathrm{~K}$.

[19] The condition of stationary phase can be expressed as $\left[\frac{\partial}{\partial k} \eta(k, x)\right]_{k=k_{0}}=0$, where $\eta(k, x)$ represents the phase of a wave packet. This expression allows one to identify where the maximum of the wave packet is situated.

[20] A. Messiah, Quantum Mechanics (Dover, New York, 1999).

[21] P. W. Milonni, The Quantum Vacuum (Academic, London, 1994).

[22] The sum over bosonic modes $j$ for our axially symmetric particle includes degenerate dipole excitations along $x$ and $y$.

[23] P. W. Milonni and R. W. Boyd, Phys. Rev. A 69, 023814 (2004).

[24] F. J. García de Abajo, Rev. Mod. Phys. 82, 209 (2010).

[25] B. T. Draine, Astrophys. J. 598, 1026 (2003).

[26] P. B. Johnson and R. W. Christy, Phys. Rev. B 6, 4370 (1972).

[27] J. D. Jackson, Classical Electrodynamics (Wiley, New York, 1999).

[28] F. Hoyle and N. C. Wickramasinghe, Mon. Not. R. Astron. Soc. 124, 417 (1962). 